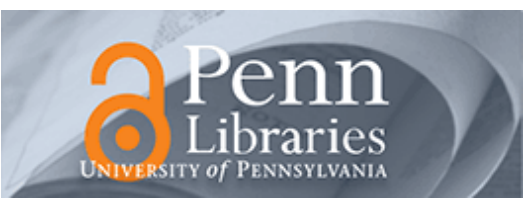

University of Pennsylvania

ScholarlyCommons

Departmental Papers (ESE)

Department of Electrical \& Systems Engineering

$5-2009$

\title{
Quantifying Acute Myocardial Injury Using Ratiometric Fluorometry
}

\author{
Mahsa Ranji \\ Burnham Institute Medical Research \\ Muneaki Matsubara \\ University of Pennsylvania
}

Bradley G. Leshnower

Emory University

Robin H. Hinmon

University of Pennsylvania, robin.hinmon@uphs.upenn.edu

Eollow this and addjtional works at: https://repository.upenn.edu/ese_papers

University of Penncylvania, jaggard@scas.upenn.cdu

\section{Recommended Citation}

Mahsa Ranii Muneaki.Matsubara Bradley G. Leshnower, Robin H. Hinmon, Dwight L. Jaggard, Britton Chance, Robert C. Gorman, and Joseph H. Gorman, "Quantifying Acute Myocardial Injury Using

Ratiometric Fluorometry", . May 2009.

Copyright 2009 IEEE. Reprinted from:

Ranji, M.; Matsubara, M.; Leshnower, B.G.; Hinmon, R.H.; Jaggard, D.L.; Chance, B.; Gorman, R.C.; Gorman, J.H., "Quantifying Acute Myocardial Injury Using Ratiometric Fluorometry," Biomedical Engineering, IEEE Transactions on , vol.56, no.5, pp.1556-1563, May 2009

URL: http://ieeexplore.ieee.org/stamp/stamp.jsp?arnumber=4663614\&isnumber=4976166

This material is posted here with permission of the IEEE. Such permission of the IEEE does not in any way imply IEEE endorsement of any of the University of Pennsylvania's products or services. Internal or personal use of this material is permitted. However, permission to reprint/republish this material for advertising or promotional purposes or for creating new collective works for resale or redistribution must be obtained from the IEEE by writing to pubs-permissions@ieee.org. By choosing to view this document, you agree to all provisions of the copyright laws protecting it.

This paper is posted at ScholarlyCommons. https://repository.upenn.edu/ese_papers/513

For more information, please contact repository@pobox.upenn.edu. 


\title{
Quantifying Acute Myocardial Injury Using Ratiometric Fluorometry
}

\begin{abstract}
Early reperfusion is the best therapy for myocardial infarction (MI). Effectiveness, however, varies significantly between patients and has implications for long-term prognosis and treatment. A technique to assess the extent of myocardial salvage after reperfusion therapy would allow for high-risk patients to be identified in the early post-MI period. Mitochondrial dysfunction is associated with cell death following myocardial reperfusion and can be quantified by fluorometry. Therefore, we hypothesized that variations in the fluorescence of mitochondrial nicotinamide adenine dinucleotide (NADH) and flavoprotein (FP) can be used acutely to predict the degree of myocardial injury. Thirteen rabbits had coronary occlusion for 30 min followed by $3 \mathrm{~h}$ of reperfusion. To produce a spectrum of infarct sizes, six animals were infused cyclosporine A prior to ischemia. Using a specially designed fluorometric probe, NADH and FP fluorescence were measured in the ischemic area. Changes in NADH and FP fluorescence, as early as 15 min after reperfusion, correlated with postmortem assessment infarct size $(r=0.695, p<0.01)$. This correlation strengthened with time $(r=0.827, p<0.01$ after $180 \mathrm{~min})$. Clinical application of catheter-based myocardial fluorometry may provide a minimally invasive technique for assessing the early response to reperfusion therapy.
\end{abstract}

\section{Keywords}

bio-optics, biomedical measurement, cardiology, cellular biophysics, diseases, fluorescence, muscle, patient treatment, acute myocardial injury, cell death, cyclosporine A, flavoprotein, fluorescence, ischemia, mitochondrial dysfunction, myocardial infarction, myocardial salvage, nicotinamide adenine dinucleotide, ratiometric fluorometry, reperfusion therapy, time $3 \mathrm{~h}$, time $30 \mathrm{~min}$, Apoptosis, fluorometery, mitochondrial disruption, mitochondrial redox state, myocardial infarction, myocardial reperfusion injury

\section{Comments}

Copyright 2009 IEEE. Reprinted from:

Ranji, M.; Matsubara, M.; Leshnower, B.G.; Hinmon, R.H.; Jaggard, D.L.; Chance, B.; Gorman, R.C.; Gorman, J.H., "Quantifying Acute Myocardial Injury Using Ratiometric Fluorometry," Biomedical Engineering, IEEE Transactions on, vol.56, no.5, pp.1556-1563, May 2009

URL: http://ieeexplore.ieee.org/stamp/stamp.jsp?arnumber=4663614\&isnumber=4976166

This material is posted here with permission of the IEEE. Such permission of the IEEE does not in any way imply IEEE endorsement of any of the University of Pennsylvania's products or services. Internal or personal use of this material is permitted. However, permission to reprint/republish this material for advertising or promotional purposes or for creating new collective works for resale or redistribution must be obtained from the IEEE by writing to pubs-permissions@ieee.org. By choosing to view this document, you agree to all provisions of the copyright laws protecting it.

\section{Author(s)}

Mahsa Ranji, Muneaki Matsubara, Bradley G. Leshnower, Robin H. Hinmon, Dwight L. Jaggard, Britton Chance, Robert C. Gorman, and Joseph H. Gorman 


\title{
Quantifying Acute Myocardial Injury Using Ratiometric Fluorometry
}

\author{
Mahsa Ranji, Student Member, IEEE, Muneaki Matsubara, Bradley G. Leshnower, Robin H. Hinmon, \\ Dwight L. Jaggard, Fellow, IEEE, Britton Chance, Life Fellow, IEEE, Robert C. Gorman, and Joseph H. Gorman, III*
}

\begin{abstract}
Early reperfusion is the best therapy for myocardial infarction (MI). Effectiveness, however, varies significantly between patients and has implications for long-term prognosis and treatment. A technique to assess the extent of myocardial salvage after reperfusion therapy would allow for high-risk patients to be identified in the early post-MI period. Mitochondrial dysfunction is associated with cell death following myocardial reperfusion and can be quantified by fluorometry. Therefore, we hypothesized that variations in the fluorescence of mitochondrial nicotinamide adenine dinucleotide (NADH) and flavoprotein (FP) can be used acutely to predict the degree of myocardial injury. Thirteen rabbits had coronary occlusion for $30 \mathrm{~min}$ followed by $3 \mathrm{~h}$ of reperfusion. To produce a spectrum of infarct sizes, six animals were infused cyclosporine A prior to ischemia. Using a specially designed fluorometric probe, NADH and FP fluorescence were measured in the ischemic area. Changes in NADH and FP fluorescence, as early as 15 min after reperfusion, correlated with postmortem assessment infarct size $(r=0.695, p<0.01)$. This correlation strengthened with time $(r=0.827, p<0.001$ after $180 \mathrm{~min})$. Clinical application of catheter-based myocardial fluorometry may provide a minimally invasive technique for assessing the early response to reperfusion therapy.
\end{abstract}

Index Terms-Apoptosis, fluorometery, mitochondrial disruption, mitochondrial redox state, myocardial infarction, myocardial reperfusion injury.

\section{INTRODUCTION}

$\mathbf{O}$ VER THE past 35 years, techniques to identify and revascularize ischemic myocardium have been developed to a

Manuscript received October 27, 2007; revised June 9, 2008. First published October 31, 2008; current version published May 22, 2009. This work was supported in part by the National Institutes of Health, Bethesda, MD, under Grant HL63954 (RCG), Grant HL76560 (JHG), Grant HL71137 (RCG), and Grant Upenn IME. Asterisk indicates corresponding author.

M. Ranji is with the Burnham Institute for Medical Research, La Jolla, CA 92037 USA.

M. Matsubara was with the Gorman Cardiovascular Research Group, Department of Surgery, School of Medicine, University of Pennsylvania, Philadelphia, PA 19036 USA. He is now with the Tsukuba University Hospital, Tsukuba 305-8577, Japan.

B. G. Leshnower is with Emory University, Atlanta, GA 30322 USA.

R. H. Hinmon and R. C. Gorman are with the Gorman Cardiovascular Research Group, Department of Surgery, School of Medicine, University of Pennsylvania, Philadelphia, PA 19036 USA (e-mail: gormanj@uphs.upenn. edu).

D. L. Jaggard is with the Complex Media Laboratory, Department of Electrical and Systems Engineering, University of Pennsylvania, Philadelphia, PA 19104 USA (e-mail: Jaggard@ seas.upenn.edu).

B. Chance is with the University of Pennsylvania, Philadelphia, PA 19104 USA.

*J. H. Gorman, III, is with the Gorman Cardiovascular Research Group, Department of Surgery, School of Medicine, University of Pennsylvania, Philadelphia, PA 19036 USA.

Digital Object Identifier 10.1109/TBME.2008.2006029 high level. Despite this, a mass epidemic of congestive heart failure (CHF) due to coronary disease has developed [1]. Five million Americans suffer from CHF with 550000 new cases diagnosed each year. Sixty-eight percent of these cases are due to coronary disease [1]. After myocardial infarction (MI), CHF is preceded by infarct expansion (i.e., the infarct stretches), progressive generalized left ventricular (LV) dilatation, and contractile dysfunction. This deleteriously progressive phenomenon has been termed postinfarction LV remodeling [2].

Once the remodeling process is established, the symptoms of CHF ensuing five-year survival, even with the most aggressive medical and surgical therapy, is about 50\% [3]. These dismal results have generated a strong interest in developing mechanical strategies for preventing infarct expansion, the resulting LV dilation, and failure.

Recent work in chronic large animal heart failure models has demonstrated that early restraint to prevent infarct stretching significantly limits ventricular dilation and preserves function [4]-[6]. Despite compelling experimental data, these techniques currently have limited clinical applicability due to an inability to identify patients early (days after infarction) at risk for remodeling. While infarct size has long been understood to correlate with the ultimate degree of ventricular remodeling [7], [8], it is a difficult parameter to quantify particularly early after reperfusion therapy.

Mitochondrial dysfunction and disruption are known to be intimately involved in both necrotic and apoptotic processes that lead to cell death following myocardial reperfusion [9], [10]. As a result, in vivo quantitative assessment of mitochondrial function may provide a method for assessing infarct size above what is currently provided by angiography and echocardiography [11], [12].

Toward this end, we have developed an optical catheter device to acquire fluorescence signals of the intrinsic mitochondrial fluorophores, nicotinamide adenine dinucleotide (NADH), and flavoprotein (FP). The ratio of these fluorescence signals $(\mathrm{FP} / \mathrm{FP}+\mathrm{NADH})$, defined as the redox ratio (RR), has been shown to correlate with different metabolic states and mitochondrial function [13], [14]. Additionally, the RR has been shown to undergo an oxidative shift in tumor cells with mitochondrial dysfunction associated with apoptosis [15]. We hypothesize that the RR within the ischemic zone, as measured by our device, can be directly correlated with infarct size early after myocardial reperfusion. Proof of such a correlation would indicate fiberoptically assessed mitochondrial fluorescence to be a quantitative tool to assess myocardial injury without invasive tissue biopsy. 


\section{Materials AND Methods}

A well-characterized rabbit model of ischemia-reperfusion injury was used [16]. In order to induce a wider range of mitochondrial disruption and myocardial injury, half the animals were treated prior to ischemia with Cyclosporin A (CsA). In this rabbit model, CsA has been shown to inhibit mitochondrial permeability transition pore opening at reperfusion following myocardial ischemia resulting in significantly less necrotic and apoptotic myocyte death [17].

The combination of within group (small differences in ischemic zone size due to varying coronary anatomy) and between group (CsA protection) provides a desirable spectrum of myocardial insults with which to correlate RR measurements.

\section{A. Surgical Protocol}

Animals are treated under experimental protocols approved by the University of Pennsylvania's Institutional Animal Care and Use Committee (IACUC) and in compliance with National Institutes of Health Publication No. 85-23, revised 1996.

Thirteen New Zealand white rabbits $(3.2-4.0 \mathrm{~kg})$ were studied in two experimental groups:

1) untreated (UnT) $(n=7): 1$-h continuous $20 \mathrm{~mL}$ infusion of saline given intravenously prior to ischemia;

2) cyclosporine $\mathrm{A}(\mathrm{CsA})$ treatment $(n=6)$ : 1 -h continuous $20 \mathrm{~mL}$ infusion of a solution of saline and CsA $(25 \mathrm{mg} / \mathrm{kg})$ given intravenously prior to ischemia.

Anesthesia was induced with intramuscular ketamine (70$100 \mathrm{mg} / \mathrm{kg})$, glycopyrrolate $(0.01 \mathrm{mg} / \mathrm{kg})$ and buprenorphine $(0.05 \mathrm{mg} / \mathrm{kg})$. After oral endotracheal intubation, animals were mechanically ventilated (Hallowell EMC Model AWS, Pittsfield, MA, USA) with air enriched with $0.6 \mathrm{~L} / \mathrm{min}$ of oxygen. Anesthesia was maintained with continuous ketamine infusion $(20 \mathrm{mg} / \mathrm{kg} / \mathrm{hr}$ IV). A high-fidelity pressure transducer (Millar Instruments Inc. Houston, TX, USA) was placed in the left ventricle via carotid artery for continuous LV pressure measurement. Peripheral arterial blood pressure, heart rate, and surface electrocardiogram (ECG) were also continuously monitored (Hewlett Packard 78534C, Palo Alto, CA, USA) and recorded (Sonometrics Inc., London, ON, Canada). Left atrial blood temperature was measured with an electrical thermometer (Thermalert TH-8 Physiotemp Instrument, Clifton, NJ, USA) and was maintained between $39^{\circ} \mathrm{C}$ to $40^{\circ} \mathrm{C}$ (normal rabbit body temperature) with a high efficiency water blanket (Medi-Therm III, Gaymar Industries Inc., Orchard Park, NY, USA).

Next, all animals received a $1-\mathrm{h}$, continuous $20 \mathrm{~mL}$ infusion of a phosphate buffered saline (PBS) vehicle (UnT), $25 \mathrm{mg} / \mathrm{kg}$ of CsA. A left thoracotomy was performed in the fourth intercostal space and the heart was exposed. A pledgetted suture (3-0 Ti-cron, U.S. Surgical, Norwalk, CT, USA) was passed around a large branch of the circumflex coronary artery at a distance $50 \%$ from the base of the heart toward the apex. Myocardial ischemia was achieved by tightening the coronary artery snare and confirmed by ST elevations on ECG and by the distinct color change of the myocardium. After $30 \mathrm{~min}$ of ischemia, the coronary artery snare was released and the myocardium was reperfused for $180 \mathrm{~min}$.

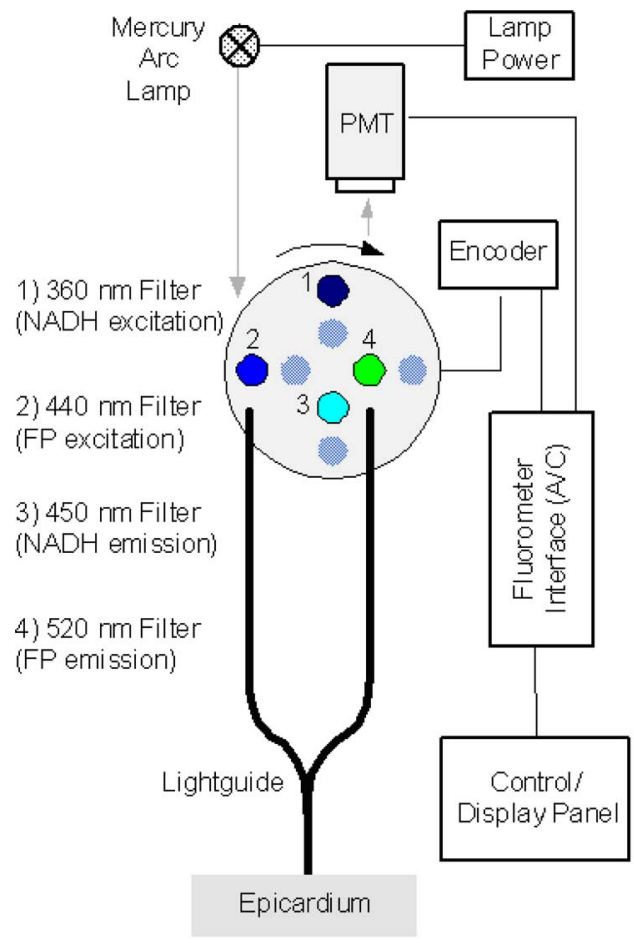

Fig. 1. Two-wavelength time-shared fluorometer in rabbit heart ratiometric measurements.

To provide a specimen of normal myocardium, one rabbit underwent the same protocol as the UnT group with the exception that no coronary was ligated and no ischemia induced.

\section{B. Myocardial Fluorescence Spectroscopy}

Fluorescence spectroscopy of rabbit myocardium in vivo was conducted with a fluorometer (Fig. 1). This fluorometer is a mobile optical-electrical apparatus that collects fluorescence signals of any type of tissue through a 3-mm-tip light guide. The incident light is a broadband mercury arc lamp that can be filtered at four different wavelengths by an air turbine filter wheel rotating at $50 \mathrm{~Hz}$. Consequently, up to four signals could be multiplexed to a photodetector in order to make fourwavelength channel optical measurements of tissue metabolism as shown in Fig. 1. In this experiment, two channels are used for excitation and the other two for emission signals. The light intensity that is incident on tissue at the fiber tip is $3 \mu \mathrm{W}$.

In cardiac fluorometry experiments, the excitation wavelengths of FP and NADH are obtained by filtering the mercury arc lamp at $436 \mathrm{~nm}$ and $366 \mathrm{~nm}$ resonance lines by interference filters 440DF20 and 365HT25 (Omega Optical, Brattleboro, VT, USA). The fluorescence intensities are then detected by a photomultiplier tube (PMT model R928, Hamamatsu, Japan), converted to an electric voltage, digitized, and displayed. A standard protocol for the fluorometer calibration is used at the beginning of each single experiment. Fluorescent standards are used from which we measure the fluorescent light at the wavelengths of interest for the upcoming experiment. We take these standard measurements into account if any adjustment factor is needed to apply to the experimental results. Specific instrument 


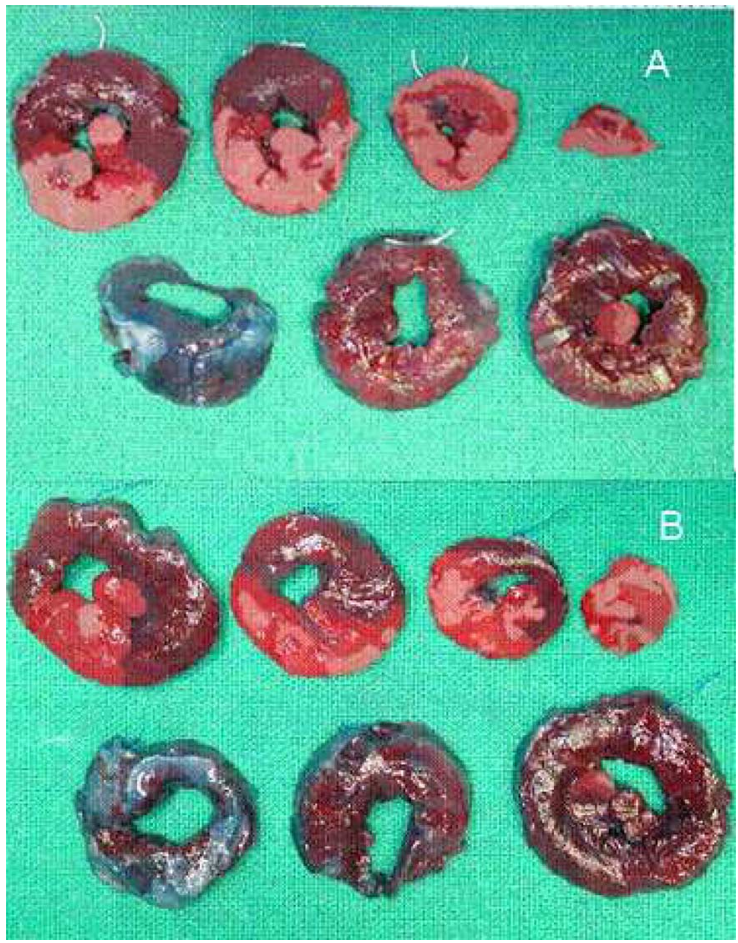

Fig. 2. Double staining technique used to identify the area at risk (AR) and the region of infarction in both the UnT group (A) and the CSA group (B). Both hearts have been sliced perpendicular to the long axis. Blue-purple myocardium has been stained by both Evans blue dye indicating it is outside the area at risk and by triphenyl-tetrazolium (TTC) indicating it is viable. Within the area at risk (no blue staining) viable myocardium has been stained dark red by TTC while the pale infracted regions have not. Notice the smaller infarct region in the CSA group.

specifications are kept the same for all the experiments (e.g. PMT high voltage, channel gain factors, etc.).

The fluorometer probe was placed on the epicardial surface in the center of the anticipated region of ischemia and continuous recording of the fluorescence signals for FP and NADH signals was performed during baseline (60 min infusion of saline or CsA), ischemia (30 min), and reperfusion (180 min). The redox ratio is calculated as $\mathrm{FP} /(\mathrm{FP}+\mathrm{NADH})$ every 5 min from the continuously recorded FP and NADH. All the redox ratio data points are then normalized by the value at time point $t=0$. These normalized redox ratios (RRs) in each group (UnT $=7$ and $\mathrm{CsA}=6$ ) are averaged and expressed as mean \pm standard deviation at 5-min time points for statistical analysis and 10minute intervals for spectroscopic graphs.

\section{Analysis of Area at Risk and Infarct Size}

At the end of the protocol, the coronary snare was reapplied, vascular clamps were used to occlude the aorta, pulmonary artery, and inferior vena cava, and the right atrium was incised. Five milliliters of Evans blue dye (1\%) (Sigma, St. Louis, MO, USA) was injected via the left atrium to delineate the ischemic myocardial risk area (AR). The heart was arrested with an intraatrial bolus of $20 \mathrm{mEq}$ of potassium chloride and was explanted. The left ventricle was sectioned perpendicular to its long axis into six to seven slices. Fig. 2 illustrates these slices stained by double staining technique for identification of area at risk. The thickness of each slice was measured with a digital micrometer and a standardized digital photograph was taken (Casio EX-Z850, Tokyo, Japan). Infarct area (I/AR) was delineated by photographing and measuring the slices after 20 min of incubation in $2 \%$ triphenyltetrazolium chloride (TTC) at $37^{\circ} \mathrm{C}$. All photographs were imported into an image analysis program (Image Pro Plus, Media Cybernetics, Silver Spring, MD, USA) and computerized planimetry was performed. The AR is expressed as a percentage of the $\mathrm{LV}$, and the infarct size is expressed as a percentage of the AR (I/AR).

\section{Transmission Electron Microscopy}

To confirm the effect of CsA on mitochondrial integrity, punch biopsies from directly under fluorometric probe were taken from two animals in the UnT group, two animals in the CsA group, and the one normal animal at the conclusion of the reperfusion protocol. Each of these biopsies was preserved in electron microscopy fixative $(2.5 \%$ glutaraldehyde, $2.0 \%$ paraformaldehyde, $0.1 \mathrm{M}$ sodium cacodylate) for $24 \mathrm{~h}$ at $4{ }^{\circ} \mathrm{C}$. After several washes in $0.1 \mathrm{M} \mathrm{NaCaC}$, samples were postfixed with buffered $2 \%$ osmium tetroxide for $1 \mathrm{~h}$ at $4^{\circ} \mathrm{C}$. Subsequent washes in $0.1 \mathrm{M} \mathrm{NaCaC}, \mathrm{H}_{2} \mathrm{O}$, and $2 \%$ aqueous uranyl acetate were then used to destain samples. Tissue samples were dehydrated in serial washes of EtOH and propylene oxide, prior to a slow infiltration with EPON 812. Finally, samples were cured at $70^{\circ} \mathrm{C}$ for $48 \mathrm{~h}$.

Cured samples were submitted to the Biomedical Imaging Core Facility at the University of Pennsylvania, where they were cut, stained, and imaged on a Jeol-10-10 transmission electron microscope (Jeol Ltd, Akishima, Japan). Random images were captured from each sample for comparative analysis. To assess the degree of mitochondrial disruption, five random images of mitochondria at $30000 \mathrm{X}$ were captured from two control, two CsA-treated, and one normal rabbit. The number of disrupted mitochondria was tallied, along with the total number of mitochondria. The percent disrupted mitochondrion (PDM) was then determined, averaged for each group, and reported.

\section{E. Statistical Analysis}

Group results for the RR, I/AR and PDM are expressed as the mean \pm standard deviation. The RR was calculated at 5 min intervals during the entire experiment for all animals. To assess between-group differences in the RR during preischemia, ischemia, and postischemia, analysis of variance (ANOVA) was used. Where ANOVA revealed significant differences, Student's $T$-test was used to compare the groups at individual time points. Postmortem values for I/AR and PDM were compared using Student's $T$-test.

At each time point, the RR was correlated with both the I/ARs using Pearson's correlation test. A $p$-value $<0.05$ was considered statistically significant. The software package used for the statistical analysis was SPSS version 11.0 for Windows (SPSS Inc., Chicago IL, USA). 


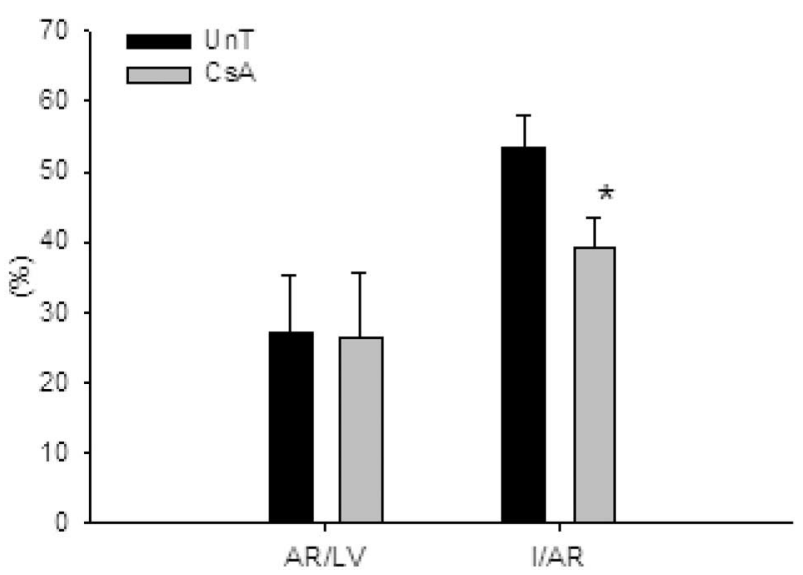

Fig. 3. Comparison of area at risk and infarct sizes between the UnT group and the CsA group. Values are means \pm standard deviation. The AR is expressed as a percentage of the $\mathrm{LV}(\mathrm{AR} / \mathrm{LV})$, and the infarct size is expressed as a percentage of the AR (I/AR). Statistically significant difference from UnT group $(p<0.01)$ is indicated by *

\section{RESULTS}

\section{A. Infarct Size Measurements}

The two-group model produced a varying degree of infarcted myocardium. The AR was similar in both groups: $27.0 \pm 8.1 \%$ in the UnT group and $26.5 \pm 9.1 \%$ in the CsA group. The I/AR was significantly smaller in the CsA group than in the UnT $(39.1 \pm 4.4 \%$ vs. $53.4 \pm 4.7 \%, p<0.0001)$ (Fig. 3).

\section{B. Mitochondrial Disruption}

The protective effect of CsA on reperfused myocardium was also quite evident in the myocardial samples studied with EM (Fig. 4). The majority of the myocytes in all three groups (one normal, two UnT, and two CsA-treated rabbits) had nuclei with a ruffled membrane and normally distributed heterochromatin. The nuclear membranes of the normal and the CsA groups were also smooth, while several abnormally shaped nuclei were found in the UnT group. Normally, the nuclear cap, or region surrounding the nucleus, is tightly packed with mitochondria. Although not as densely packed with mitochondria as the normal group, tightly packed mitochondria were still present in the nuclear caps of the CsA group. Most mitochondria of the CsAtreated animals had well-defined outer membranes. Although a small percentage of the mitochondria had dilated cristae, the majority of the CsA cristae exhibited normal characteristics. The nuclear caps of the UnT group were either absent of mitochondria or possessed mitochondria with disrupted outer membranes and tightly packed cristae, vacuolated mitochondria with poorly defined outer membranes, or mitochondria absent of an outer membrane and unraveling cristae. For all three groups, the mitochondria between the muscle fibers possessed characteristics similar to what was found in the nuclear cap of the respective group. The percent disrupted mitochondria for the UnT group was $53.31 \% \pm 16.47 \%$, for the CsA group, $19.71 \% \pm 9.64 \%$, and for the normal group $1.65 \%$ (Fig. 5).

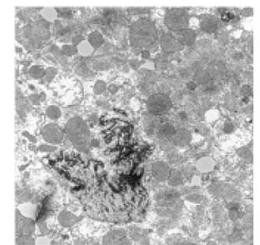

(A)

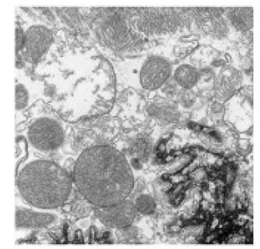

(D)

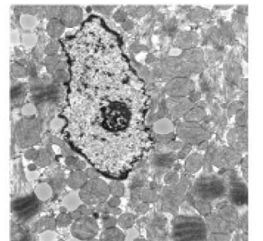

(B)

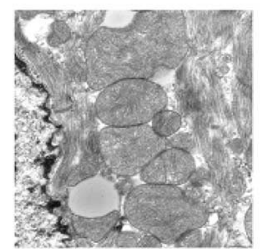

(E)

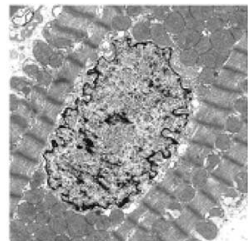

(C)

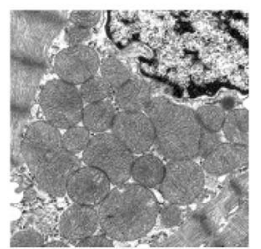

(F)
Fig. 4. Comparison of mitochondrial changes in the electron microscopy imaging between the UnT group, the CsA group, and normal group. Normally, the nuclear cap, or region surrounding the nucleus, is tightly packed with mitochondria $(\mathrm{C}, \mathrm{F})$. Although not as densely packed with mitochondria as the normal group, tightly packed mitochondria were still present in the nuclear caps of the CsA group (B). Most mitochondria of the CsA-treated animals had welldefined outer membranes. Although a small percentage of the mitochondria had dilated cristae, the majority of the CsA cristae exhibited normal characteristics (E). The nuclear caps of the UnT group were either absent of mitochondria or possessed mitochondria with disrupted outer membranes and tightly packed cristae, vacuolated mitochondria with poorly defined outer membranes, or mitochondria absent of an outer membrane and unraveling cristae (A, D). For all three groups, the mitochondria between the muscle fibers possessed characteristics similar to what was found in the nuclear cap of the respective group. (A) UnT-12000X. (B) CsA-12000X. (C) Normal-12000X. (D) UnT-30000X. (E) CsA-30000X. (F) Normal-30000X.

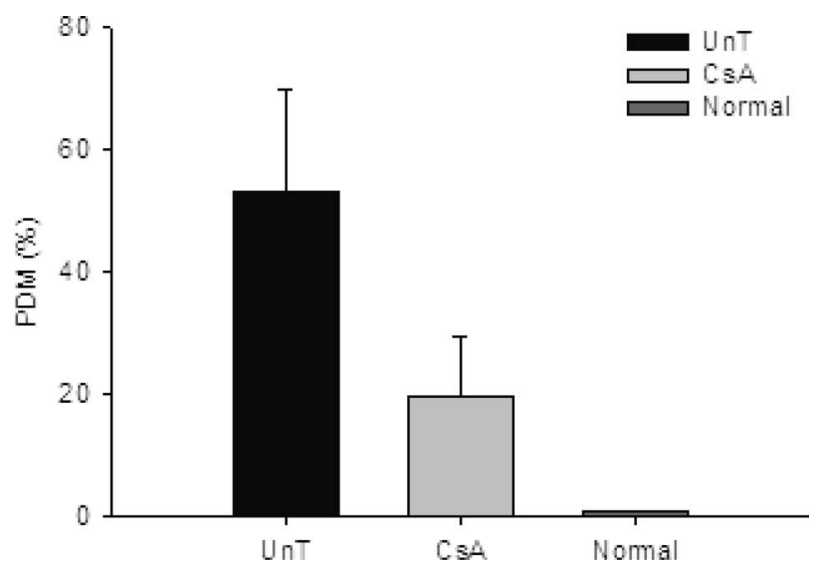

Fig. 5. Comparison of the percent disrupted mitochondria (PDM) between the UnT group, the CsA group, and normal group. Values are means \pm standard deviation. PDM = percent disrupted mitochondria.

\section{Redox Fluorometry}

Fig. 6 presents the RR at 10 min intervals during the entire protocol. The RR remained constant in both groups during the pre-ischemic $\mathrm{CsA} /$ saline infusion period demonstrating that CsA had no effect on the mitochondrial metabolic state of normally perfused myocardium.

In both groups, the RR drops immediately with the onset of ischemia and continues to drop steadily during the entire $30 \mathrm{~min}$. This is consistent with marked reduction in the oxidative state of the fluorophores due to decreased oxygen availability. That is, NADH and FP remain in their reduced form due to backup of 


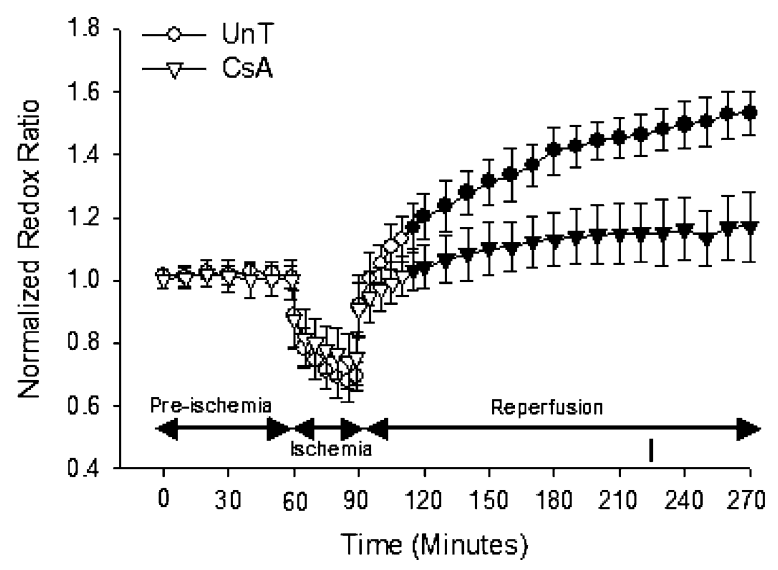

Fig. 6. Comparison of mean normalized redox ratio between the UnT group and CsA group throughout the experiment. Statistically significant difference between the UnT and CsA groups $(p<0.05)$ is indicated by filled circle and triangle. Error bars represent twice the standard deviation.

TABLE I

CORRELATIONS BETWEEN RR AND I/AR AFTER REPERFUSION

\begin{tabular}{|c|c|c|}
\hline Minutes of reperfusion & $\mathbf{r}$ & p value \\
\hline \hline 0 & 0.248 & 0.4132 \\
\hline 15 & 0.695 & $0.0084^{*}$ \\
\hline 30 & 0.792 & $0.0012^{*}$ \\
\hline 45 & 0.792 & $0.0012^{*}$ \\
\hline 60 & 0.807 & $0.0008^{*}$ \\
\hline 75 & 0.822 & $0.0006^{*}$ \\
\hline 90 & 0.861 & $0.0002^{*}$ \\
\hline 105 & 0.863 & $0.0001^{*}$ \\
\hline 120 & 0.865 & $0.0001^{*}$ \\
\hline 135 & 0.851 & $0.0002^{*}$ \\
\hline 150 & 0.838 & $0.0004^{*}$ \\
\hline 165 & 0.811 & $0.0008^{*}$ \\
\hline 180 & 0.827 & $0.0005^{*}$ \\
\hline
\end{tabular}

Statistically significant difference $(p<0.01)$ is indicated by *

the electron transport chain. The rate at which the RR decreased was slightly less in the CsA group (Fig. 6), but this trend did not reach statistical significance.

With the reinstitution of blood flow, the RR increased immediately in both groups. In the UnT group, the RR increased persistently by $53.1 \pm 6.8 \%$ over normal during the $180 \mathrm{~min}$ of reperfusion. This drastic increase in the RR represents a "hyper-oxidation" of fluorophores and is indicative of dysfunctional mitochondria that are unable to reduce $\mathrm{NAD}^{+}$and FP thus preventing their entry into the electron transport chain and ultimately the production of adenosine triphosphate. In the CsA group, the RR ratio only increases by $17.0 \pm 11.0 \%$ of normal demonstrating less mitochondrial dysfunction.

During the first $15 \mathrm{~min}$ after reperfusion the RR increases almost linearly in both groups but with a much greater slope in the UnT group. After 15 min of reperfusion, the RR of the UnT group is significantly greater than that of the CsA group. After 30 min, the RR changes very little in the CsA group.

Table I demonstrates the correlation between the RR and I/AR at 15-min time intervals after reperfusion. It can be seen that as early as $15 \mathrm{~min}$ after reperfusion, the $\mathrm{RR}$ ratio correlates

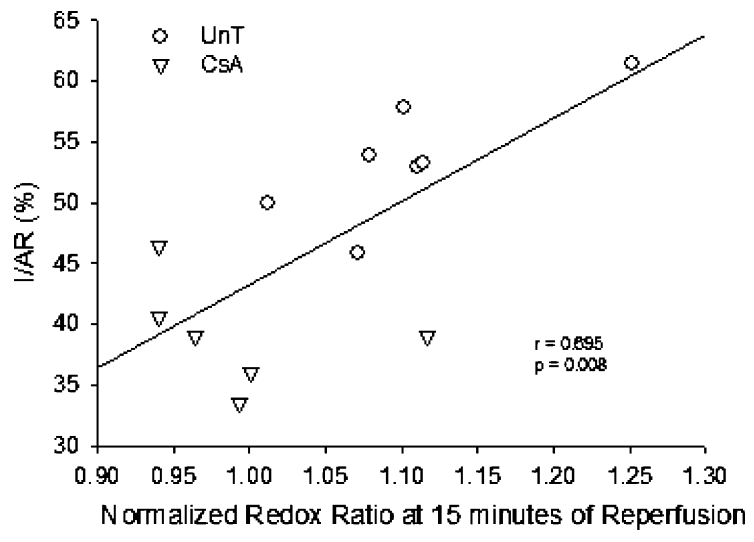

Fig. 7. Plot of infarct size versus normalized redox ratio at $15 \mathrm{~min}$ of reperfusion in the UnT group and the CsA group.

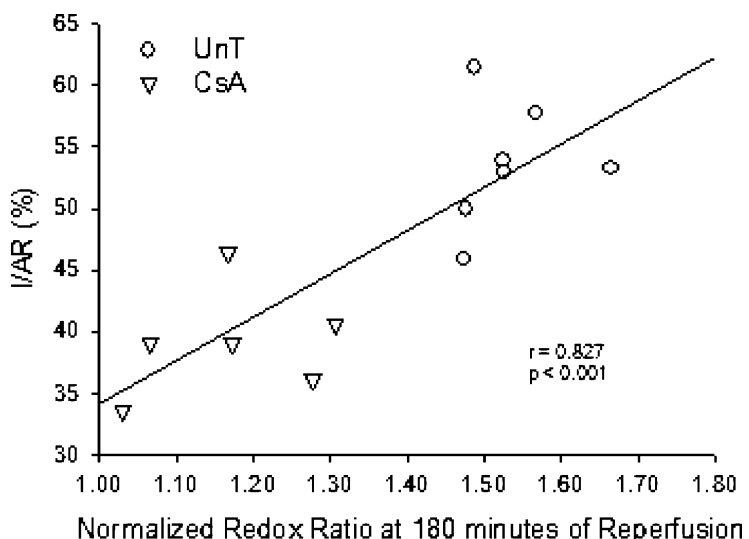

Fig. 8. Plot of infarct size versus normalized redox ratio at $180 \mathrm{~min}$ of reperfusion in the UnT group and the CsA group.

strongly with infarct size. Figs. 7 and 8 are scatter plots of the RR versus the I/AR that demonstrate the predictive strength of the correlation at 15 and 180 min after reperfusion, respectively.

\section{DISCUSSION}

Mitochondrial permeability transition (MPT) pore opening is recognized as a pivotal event in necrotic and apoptotic cell death [18], [19]. Following myocardial ischemia-reperfusion injury, opening of this nonspecific pore results in inner membrane potential collapse, uncoupling of the respiratory chain, and efflux of small molecules such as cytochrome $c$ and other proapoptotic factors [20]. CsA is a powerful inhibitor of the MPT pore, and several reports indicate that it protects the isolated heart from ischemia-reperfusion injury [17]. In this study, the protective role of CsA was confirmed in vivo and exploited to provide an experimental model with a spectrum of reperfusioninduced mitochondrial dysfunction and resulting myocardial injury. This model was used to test the hypothesis that changes in mitochondrial NADH and FP fluorescence can be measured and correlated with myocardial injury.

Our data indicates that myocardial reperfusion injury is associated with mitochondrial disruption, which is significantly blunted by CsA, and that this injury correlates very strongly 
with the RR as measured by fluorometry. This is particularly compelling considering that the fluorometric measurements of NADH and FP were made over a relatively small epicardial region of the area at risk. We found it equally interesting that even very early after reperfusion ( $15 \mathrm{~min}$ ), the RR began to correlate with infarct size at $3 \mathrm{~h}$ postreperfusion.

These data provide convincing evidence that fluorometry can be used to clinically assess myocardial injury noninvasively without tissue biopsy. Such a tool will provide a better early evaluation of the effectiveness of reperfusion therapy and potentially identify patients that are at increased risk for postinfarction ventricular remodeling. Early identification of such patients would allow the institution of more aggressive infarct restraint procedures to prevent heart failure than would be justifiable without proper patient identification.

Apoptotic-induced mitochondrial dysfunction is associated with established chronic heart failure [21] as well as myocardial rejection after heart transplantation [22]. Both these disease states are currently treated with complex pharmacological strategies that can be hard to optimize. Considering the findings of this study, it is possible that fluorometry may be used to assess and monitor the progression of these diseases without tissue biopsy and allow physicians to make more informed decisions regarding drug selection and dosing.

To prove the clinical applicability of this technology, we are currently in the process of designing human experiments in which the redox ratio will be correlated with mitochondrial disruption as measured by electron microscopy in cardiovascular specimens. The first human experiments will be conducted on patients with end-stage heart failure requiring placement of a ventricular assist device (VAD). During VAD placement, a portion of the failing LV is routinely removed. Prior to excision of the myocardium, the redox ratio will be measured. The myocardium will then be removed and assessed for mitochondrial disruption using the electron microscopic techniques described in this manuscript and the two measurements correlated.

While development hurdles persist, one can envision a catheter-based fluorometer that will be introduced via standard percutaneous venous or arterial techniques to assess the myocardial injury associated with reperfusion, ventricular remodeling, and allograft rejection. We believe that in human application, transvascular approaches will allow the fluorometric catheter to be placed directly on the tissue of interest and that signal quality will be very similar to those reported in this manuscript. Such a technology would improve the care of these very complex patients while at the same time minimizing their discomfort and treatment risk.

\section{ACKNOWLEDGMENT}

M. Ranji and M. Matsubara have contributed equally to the preparation of this manuscript.

\section{REFERENCES}

[1] M. Gheorghiade and R. O. Bonow, "Chronic heart failure in the United States: A manifestation of coronary artery disease," Circulation, vol. 97, no. 3, pp. 282-289, Jan. 1998.
[2] J. Narula, M. S. Dawson, B. K. Singh, A. Amanullah, E. R. Acio, F. A. Chaudhry, R. B. Arani, and A. E. Iskandrian, "Noninvasive characterization of stunned, hibernating, remodeled and nonviable myocardium in ischemic cardiomyopathy," J. Amer. Coll. Cardiol., vol. 36, no. 6, pp. 1913-1918, Nov. 2000.

[3] A. Yamaguchi, T. Ito, H. Adachi, S. Murata, H. Kamio, M. Okada, and J. Tsuboi, "Left ventricular volume predicts postoperative course in patients with ischemic cardiomyopathy," Ann. Thorac. Surg., vol. 65, no. 2, pp. 434-438, Feb. 1998.

[4] A. S. Blom, R. Mukherjee, J. J. Pilla, A. S. Lowry, W. M. Yarbrough, J. T. Mingoia, J. W. Hendrick, R. E. Stroud, J. E. McLean, J. Affuso, R. C. Gorman, J. H. Gorman, III, M. A. Acker, and F. G. Spinale, "Cardiac support device modifies left ventricular geometry and myocardial structure after myocardial infarction," Circulation, vol. 112, no. 9, pp. 1274-1283, Aug. 2005.

[5] J. J. Pilla, A. S. Blom, J. H. Gorman, III, D. J. Brockman, J. Affuso, L. M. Parish, H. Sakamoto, B. M. Jackson, M. A. Acker, and R. C Gorman, "Early postinfarction ventricular restraint improves borderzone wall thickening dynamics during remodeling," Ann. Thorac. Surg., vol. 80, no. 6, pp. 2257-2262, Dec. 2005.

[6] Y. Enomoto, J. H. Gorman, III, S. L. Moainie, B. M. Jackson, L. M. Parish T. Plappert, A. Zeeshan, and M. G. St. John-Sutton, R. C. Gorman, "Early ventricular restraint after myocardial infarction: Extent of the wrap determines the outcome of remodeling," Ann. Thorac. Surg., vol. 79, no. 3, pp. 881-887, Mar. 2005 .

[7] R. G. McKay, M. A. Pfeffer, R. C. Pasternak, J. E. Markis, P. C. Come, S. Nakao, J. D. Alderman, J. J. Ferguson, R. D. Safian, and W. Grossman, "Left ventricular remodeling after myocardial infarction: A corollary to infarct expansion," Circulation, vol. 74, no. 4, pp. 693-702, Oct. 1986.

[8] J. A. Erlebacher, J. L. Weiss, L. W. Eaton, C. Kallman, M. L. Weisfeldt, and B. H. Bulkly, "Late effects of acute infarct dilatation on heart size: A two dimensional echocardiographic study," Amer. J. Cardiol., vol. 49, no. 5, pp. 1120-1126, Apr. 1982.

[9] R. A. Gottlieb, K. O. Burleson, R. A. Kloner, B. M. Babior, and R. L. Engler, "Reperfusion injury induces apoptosis in rabbit cardiomyocytes," J. Clin. Invest, vol. 94, no. 4, pp. 1621-1628, Oct. 1994.

[10] H. J. Bromme and J. Holtz, "Apoptosis in the heart: When and why?," Mol. Cell. Biochem., vol. 261-275, pp. 163-164, Oct.-Nov. 1996.

[11] H. L. Wyatt, S. Meerbaum, M. K. Heng, J. Rit, P. Gueret, and E. Corday, "Experimental evaluation of the extent of myocardial dyssynergy and infarct size by two-dimensional echocardiography," Circulation, vol. 63, no. 3, pp. 607-614, Mar. 1981.

[12] N. G. Pandian, S. Koyanagi, D. J. Skorton, S. M. Collins, C. L. Easthm, R. A. Kieso, M. L. Marcus, and R. E. Kerber, "Relations between 2dimensional echocardiographic wall thickening abnormalities, myocardial infarct size and coronary risk area in normal and hypertrophied myocardium in dogs," Amer. J. Cardiol., vol. 52, no. 10, pp. 1318-1325, Dec. 1983.

[13] B. Chance and H. Baltscheffsky, "Respiratory enzymes in oxidative phosphorylation. VII. Binding of intramitochondrial reduced pyridine nucleotide," J. Biol. Chem., vol. 233, no. 3, pp. 736-739, Sep. 1958.

[14] B. Chance and B. Schoener, "High and low energy states of cytochromes. II. In submitochondrial particles," J. Biol. Chem., vol. 241, no. 20, pp. 4577-4587, Oct. 1966.

[15] M. Ranji, S. Kanemoto, M. Matsubara, M. A. Grosso, J. H. Gorman III, R. C. Gorman, D. L. Jaggard, and B. Chance, "Fluorescence spectroscopy and imaging of myocardial apoptosis," J. Biomed. Opt., vol. 11, no. 6, pp. 064036-1-064036-4, Nov.-Dec. 2006.

[16] B. G. Leshnower, H. Sakamoto, A. Zeeshan, L. M. Parish, R. Hinmon, T. Plappert, J. H. Gorman III,, and R. C. Gorman, "Role of acetaminophen in acute myocardial infarction," Amer. J. Physiol. Heart Circ. Physiol., vol. 81, no. 4, pp. 2424-2431, Jun. 2006.

[17] L. Argaud, O. Gateau-Roesch, D. Munteam, L. Chalabreysse, J. Loufouat, D. Robert, and M. Ovize, "Specific inhibition of the mitochondrial permeability transition prevents lethal reperfusion injury," J. Mol. Cell. Cardiol, vol. 38, no. 2, pp. 367-374, Feb. 2005.

[18] G. Kroemer, B. Dallaporta, and M. Resche-Rignon, "The mitochondrial death/life regulator in apoptosis and necrosis," Annu. Rev. Physiol., vol. 60, pp. 619-642, 1998.

[19] M. R. Duchen, O. McGuiness, L. A. Brown, and M. Crompton, "On the involvement of a cyclosporin a sensitive mitochondrial pore in myocardial reperfusion injury," Cardiovasc. Res., vol. 27, no. 10, pp. 1790-1794, Oct. 1993.

[20] M. Zoratti and I. Szabo, "The mitochondrial permeability transition," Bichim. Biophys. Acta, vol. 1241, no. 2, pp. 139-176, Jul. 1995. 
[21] J. Narula, N. Haider, R. Virmani, T. G. DiSalvo, F. D. Kolodgie, R. J. Hajjar, U. Schmidt, M. J. Semigran, G. W. Dec, and B. A. Khaw, "Apoptosis in myocytes in end-stage heart failure," N. Engl. J. Med., vol. 335, no. 16, pp. 1182-1189, Oct. 1996.

[22] J. Narula, E. R. Acio, N. Narula, L. E. Samuels, B. Fyfe, D. Wood, J. M. Fitzpatrick, P. N. Raghunath, J. E. Tomaszewski, C. Kelly, N. Steinmetz, A. Green, J. F. Tait, J. Leppo, F. G. Blankenberg, D. Jain, and H. W. Strauss, "Annexin-V imaging for noninvasive detection of cardiac allograft rejection," Nat. Med., vol. 7, no. 12, pp. 1347-1352, Dec. 2001.

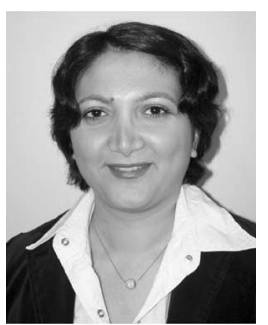

Mahsa Ranji (S'06) received the B.Sc. degree from Sharif University, Tehran, Iran, in 1988, the M.Sc. degree from Khajeh Nasir Toosi University, Tehran, Iran, in 2000, and the Ph.D. degree in electrical and systems engineering from the University of Pennsylvania, Philadelphia, in December 2007, under the supervision of Prof. Chance and Prof. Jaggard in collaboration with Gorman laboratory.

She has currently started postdoctoral research in fluorescence studies of stem cells at the Burnham Institute for Medical Research/University of California San Diego Bioengineering, La Jolla, CA. Her current research interests include fluorescence spectroscopy and imaging for biomedical applications. Particularly, she has collaborated with the Surgery Department, University of Pennsylvania for developing optical techniques for diagnosis of myocardial infarction (heart attack) and its possible clinical transferability.

Dr. Ranji is a member of WIE, the Optical Society of America, and the International Society for Optical Engineering.

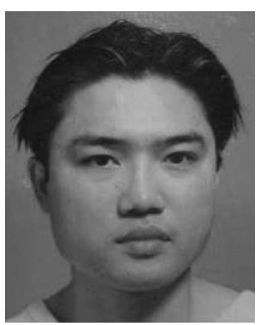

Muneaki Matsubara received the M.D. degree, in 1998, from Tsukuba University School of Medicine, Tsukuba, Japan.

During 2005-2007, he was with the Gorman Cardiovascular Research Group, University of Pennsylvania, where he was engaged in research on myocardial ischemia/reperfusion injury. He is currently a Chief Resident in cardiothoracic surgery at the Tsukuba University Hospital, Tsukuba. His current research interests include the pathophysiology and treatment of heart failure, ventricular assist device design, percutaneous valve repair/replacement and cardiac imaging.

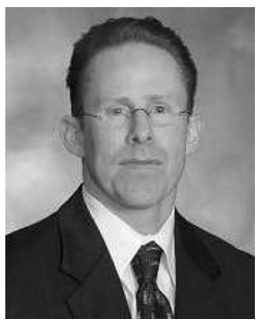

Bradley G. Leshnower received the B.S. degree in cello performance from the University of Texas at Austin, Austin, and the M.D. degree from the University of Texas Southwestern Medical School, Austin.

He completed the general surgery training at the University of Pennsylvania, where he was also a Research Fellow with the Gorman Cardiovascular Research Group. He is currently a Resident in cardiothoracic surgery at Emory University, Atlanta, GA. His current research interests include ischemia/reperfusion injury.

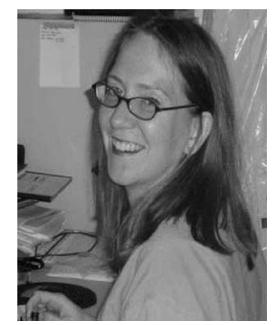

Robin H. Hinmon received the B.A. degree in biology education, in 1997, from the University of Delaware Honors Program, Newark. She is currently working toward the M.S. degree at the University of the Sciences in Philadelphia, Philadelphia, PA, where she was engaged in characterizing the taxonomic relationship among Agrobacterium tumefaciens, A. rhizogenes, and Rhizobium leguminosarum.

In 2002, she accepted an immunohistochemistry research position at the University of Pennsylvania in the Laboratory of Robert C. Gorman, M.D., and Joseph H. Gorman, III, M.D., where large and small animal models were used to characterize heart failure and valve disease. She enriched the pathophysiology studies by introducing immunofluorescence, confocal microscopy, and transmission electron microscopy. She currently manages all laboratory research for the Gorman Cardiovascular Research Group. Her studies are focused on characterizing apoptosis in the heart from a molecular, protein, and pathophysiological viewpoint.

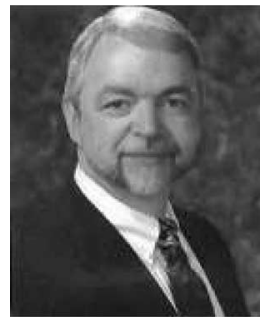

Dwight L. Jaggard (S'68-M'77-SM'86-F'91) was born in Oceanside, NY. He received the B.S.E.E. and M.S.E.E. degrees from the University of Wisconsin, Madison, in 1971 and 1972, respectively, and the $\mathrm{Ph} . \mathrm{D}$. degree in electrical engineering and applied physics from California Institute of Technology (Caltech), Pasadena, in 1976.

From 1976 to 1978, he was a Postdoctoral Research Fellow at Caltech and a Consultant to the Jet Propulsion Laboratory. In 1978, he joined the faculty at the University of Utah, Salt Lake City, as an Assistant Professor of electrical engineering. Since 1980, he has been engaged in research on complex media, scattering and inverse scattering, and highresolution imaging at the Moore School of Electrical Engineering, University of Pennsylvania, Philadelphia. He was an Associate Dean for graduate education and research in the School of Engineering and Applied Science from 1992 through 1999, and is currently a Professor of systems and electrical engineering and Director of the Executive Master's of Technology Management Program at the University of Pennsylvania, where he co-founded the Complex Media Laboratory in the Department of Electrical and Systems Engineering. He is the Co-Editor of and contributor to the book Recent Advances in Electromagnetic Theory (Springer-Verlag, 1990), and is a contributor to the books Symmetry in Electromagnetics (Taylor and Francis, 1995), Fractals in Engineering (Springer, 1997), Fractals: Theory and Applications in Engineering (Springer, 1999), and Frontiers of Electromagnetics (IEEE Press, 2000). He holds a patent. His current research interests include characteristics and applications of fractal electrodynamics to scattering and radiation, problems in inverse scattering; applications of biooptics, and the effects of knot symmetry and topology on waves.

Prof. Jaggard is a Fellow of the Optical Society of America, and a member of the Electromagnetics Academy. He has served as an Editor of the Journal of Electromagnetic Wave Applications and Editor of several special sections of the Journal of the Optical Society and the PROCEEDINGS OF THE IEEE regarding fractals and their applications. He was a Member of the Editorial Board of the PROCEEDINGS OF THE IEEE. He was the recipient of numeous awards including the S. Reid Warren Award for Distinguished Teaching, the Christian F. and Mary R. Lindback Award for Distinguished Teaching, and the EMTM Award for Excellence in Teaching.

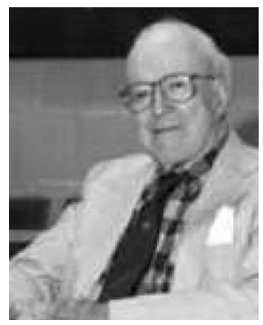

Britton Chance (M'46-SM'46-F'54-LF'81) received the $\mathrm{Ph} . \mathrm{D}$. degree in physical chemistry, in 1940, from University of Pennsylvania, Philadelphia, and the Ph.D. degree in biology and physiology in 1942 and the honorary D.Sc. degree ten years later, both from Cambridge University, Cambridge, U.K.

$\mathrm{He}$ became a member of the University of Pennsylvania faculty in 1941 as an Assistant Professor of biophysics and physical biochemistry in the School of Medicine. He served as a Professor of biophysics and physical biochemistry from 1949 until 1983 in the School of Medicine, University of Pennsylvania. He was also the Director of the E. R. Johnson Foundation in the School of Medicine for this entire period. Since 1983, he has continued at the University of Pennsylvania and is currently the Eldridge Reeves Johnson University Professor Emeritus of biophysics, physical chemistry, and radiologic physics. He is also a Senior Visiting Scientist at the Singapore Bioimaging Consortium, Helios, Singapore. $\mathrm{He}$ has made contributions not only in the identification and functioning of enzyme-substrate compounds, but also in areas of magnetic resonance spectroscopy in humans, the mathematics of the inverse problem, light transport in highly scattering media, breast cancer diagnostics, and muscle dynamics. He has been a pioneer in the field of biomedical optics, including the efforts to develop spectroscopy as a noninvasive analytical tool for clinical diagnosis. $\mathrm{He}$ is the author or coauthor of more than 2000 original articles, chapters and reviews. He has mentored over 300 graduate students and postdocs. His current research interests include mitochondrial metabolism and tissue oxygenation. $\mathrm{He}$ has developed spectroscopic and imaging methods for breast cancer detection, cognitive function, myocardium redox states, and muscle function.

Prof. Chance is a Fellow of the Optical Society of America. He was elected to the National Academy of Science in 1954. He was the recipient of numerous awards including the Olympic Gold Medal for the U.S. Yacht team in 1952, the Pennsylvania Award for Excellence (Life Sciences), in 1968, the National Medal of Science in 1974, and the Lifetime Achievement Award from the International Society for Optical Engineering in 2005. 


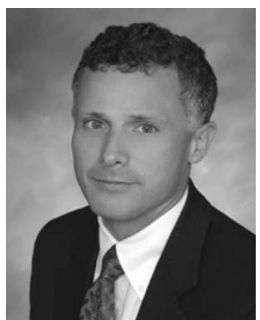

Robert C. Gorman received the B.S. degree (summa cum laude) in chemical engineering from the New Jersey Institute of Technology, Newark, NJ, and the M.D. degree from the University of Medicine and Dentistry of New Jersey, Stratford, NJ.

He completed residencies in general surgery and cardiothoracic surgery at the Hospital of the University of Pennsylvania and Children's Hospital of Philadelphia. While a resident, he completed a twoyear National Institutes of Health funded postdoctoral fellowship in the laboratory of Henry Edmunds during which he did groundbreaking research to elucidate the mechanism of ischemic/functional mitral regurgitation. He is currently an Associate Professor of surgery and Director of Cardiac Surgical Research, Gorman Cardiovascular Research Group, University of Pennsylvania, Philadelphia. He is board certified in both general and cardiothoracic surgery. His clinical focus is on adult cardiac surgery with a particular emphasis on valvular heart disease, aortic disease, ventricular assist devices, heart and lung transplantation, and heart failure. $\mathrm{He}$ also directs a heart failure and device design laboratory. He is the author or coauthor of more than 130 original articles, chapters, and reviews. He has mentored over 20 postdoctoral fellows, medical students, and undergraduates. His current research interests include the pathophysiology and treatment of heart failure, ventricular assist device design, percutaneous valve repair/replacement, cardiac imaging with MRI, CT and real-time 3-D echocardiography, mitral valve physiology, ventricular pacing for heart failure, valve calcification, and biocompatibility of synthetic surfaces. He has served on numerous study sections and advisory committees for both the National Institutes of Health and the American Heart Association.

Dr. Gorman has recently been the recipient of a prestigious Established Investigator Award from the American Heart Association.

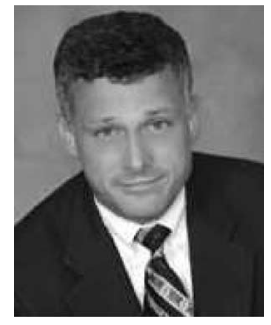

Joseph H. Gorman, III, received the B.S. degree (summa cum laude) in chemical engineering from the New Jersey Institute of Technology, Newark, NJ, and the M.D. degree (with honors) from the University of Medicine and Dentistry of New Jersey, Stratford, NJ

He completed residencies in general surgery and cardiothoracic surgery at the Hospital of the University of Pennsylvania., Philadelphia. While a resident, he completed a two-year National Institutes of Health funded postdoctoral fellowship in the laboratory of Henry Edmunds during which he did groundbreaking research in the field of cardiac remodeling, mitral valve disease, and cardiac imaging. He currently codirects the Gorman Cardiovascular Research Group, University of Pennsylvania. $\mathrm{He}$ is board certified in both general and cardiothoracic surgery. In addition to his research activities, he also performs an extensive amount of esophageal surgery for malignant and benign disease. He also directs a heart failure and device design laboratory. He is the author or coauthor of more than 100 original articles, chapters, and reviews. He has mentored over 20 postdoctoral fellows, medical students, graduate students, and undergraduates. His current research interests include the pathophysiology and treatment of heart failure, ventricular assist device design, percutaneous valve repair/replacement, cardiac imaging with MRI, CT and real-time 3-D echocardiography and near-IR spectroscopy, mitral valve physiology, ventricular pacing for heart failure, valve calcification, and biocompatibility of synthetic surfaces. He has served on numerous study sections and advisory committees for both the National Institutes of Health and the American Heart Association. 